\title{
Absorption and Scattering Microscopy of Single Metal Nanoparticles.
}

\author{
M. A. van $\operatorname{Dijk}^{a}$, A. L. Tchebotareva ${ }^{a}$, M. Orrit ${ }^{a *}$ \\ a MoNOS, Huygens Laboratory, University of Leiden \\ P. O. Box 9504, 2300 RA Leiden, The Netherlands \\ M. Lippitz ${ }^{b}$ \\ ${ }^{b}$ Institute for Physical Chemistry, Johannes Gutenberg University of Mainz, \\ Jakob-Welder-Weg 11, D-55099 Mainz, Germany

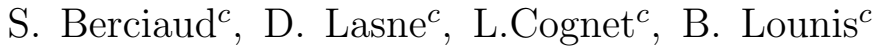 \\ c Université Bordeaux, 351, Cours de la Libération, 33405 Talence Cedex, France
}

(Dated: May 18, 2006)

\begin{abstract}
Several recently developed detection techniques opened studies of individual metal nanoparticles (1-100 $\mathrm{nm}$ in diameter) in the optical far field. Eliminating averaging over the broad size and shape distributions produced by even the best of current synthesis methods, these studies hold great promise for gaining a deeper insight into many of the properties of metal nanoparticles, notably electronic and vibrational relaxation. All methods are based on detection of a scattered wave emitted either by the particle itself, or by its close environment. Direct absorption and interference techniques rely on the particle's scattering and have similar limits in signal-to-noise ratio. The photothermal method uses a change in the refractive index of the environment as an additional step to enhance the scattered wave. This leads to a considerably improved sensitivity. We briefly discuss and compare these various techniques, review the new results they generated so far, and conclude on their great potential for nanoscience and for single-molecule labelling in biological assays and live cells.
\end{abstract}

\footnotetext{
*E-mail:orrit@molphys. leidenuniv.nl
} 


\section{INTRODUCTION}

Nanoparticles, i.e. solid particles with sizes ranging from a few nanometers to several hundreds of nanometers, have properties that differ significantly from those of bulk materials [1]. Metal nanoparticles attract strong interest both because they open up a new field in fundamental science and because of their potential technological applications. They are convenient components for sub-wavelength optical devices [2-5], for nonlinear optics [68], for optical data storage [9], for surface-enhanced spectroscopy [10] and catalysis [11], and for biological labelling and sensing $[12,13]$. The unique optical properties of metallic nanoparticles arise from the large density and susceptibility of their free electrons. The collective particle plasmon mode strongly interacts with optical waves. Traditionally, optical studies of metal nanoparticles (studies of optical absorption or scattering [14-16], non-linear optical properties [17], luminescence [18, 19], structural and vibrational properties [15, 16, 20,21]) have been performed on large ensembles. Studying isolated single particles eliminates a problem always present in ensemble measurements: the averaging over a distribution of sizes, shapes, and structural defects such as interfaces, as well as possible interaction effects between particles when their concentration is not sufficiently low.

Nano-optics and plasmonics, i.e., studies of the local field around a particle, of the interaction between particles, and more generally of the field distribution in the immediate vicinity of metallic nanostructures, demand a spatial resolution beyond the optical diffraction limit. Hence, near-field optical techniques are natural for studying particle plasmon interactions and field enhancement at sub-wavelength scales, as shown in recent studies $[22,23]$. However, as soon as the inter-particle distance is large compared to the optical wavelength, far-field optical methods can address individual particles in an experimentally less demanding way, while still providing valuable information.

The general theory of light scattering by a spherical particle was developed by G. Mie [24, 25]. The scattering cross-section of a particle of radius $R$ much smaller than the wavelength $\lambda$ of the light (or rather with $2 \pi R \ll \lambda$ ), varies as $R^{6}$, while its absorption cross-section varies as $R^{3}$ only. Therefore, for very small particles absorption is more important than scattering, and absorption-based detection methods are more sensitive. Scattering becomes more important than absorption when the circumference of the particle is comparable to the wavelength of light. For example, for Au spheres in water probed by 532-nm laser light, 
absorption is more important than scattering for diameters below $100 \mathrm{~nm}$ (Figure 1).

There are several approaches to the far-field optical detection of individual metal nanoparticles. They are based on either the scattered intensity [26, 27], or on the generation of new wavelengths by the particle, either in a linear photoluminescence process [28-30] or in nonlinear processes $[6,31,32]$. A general review of these methods was recently published [33]. In the present article, we focus on optical studies of single metal nanoparticles in which detection is done at the same wavelength as the one used for excitation (or for probing, in the case of the photothermal or pump-probe methods). In all current approaches, one uses directly or indirectly the interference of the scattered wave with a reference wave. The advantage of the interference signal is that it varies only with the third power of the particle size. Interferometry not only improves the sensitivity of both absorption- and scattering-based methods, but it can also give access to both the amplitude and the phase of the scattered wave. Short laser pulses can be used to probe the time-dependent optical response of metal nanoparticles. Combination of high temporal or spectral resolution with the ultimate spatial resolution at the single-particle level, gives a new insight into the electronic relaxation processes [34, 35] of nanoparticles, as well as into their vibrational properties [36].

The paper is organized as follows: A short overview of optical properties of metal nanoparticles and of optical detection principles is given in Section II. Section III reviews the experimental absorption and scattering techniques based on the direct response of the nanoparticle and summarizes the main experimental results obtained with these methods. In Section IV we review photothermal methods which are based on a detection of the refractive index changes of the particle's surrounding, resulting from particle heating by an auxiliary pump beam. These methods can also be applied to other absorbing nanoobjects. Section V concludes the paper.

\section{GENERAL THEORETICAL CONSIDERATIONS}

Interaction of light with a metal object may, under suitable conditions, result in an excitation of a surface-bound wave of electron density, known as surface plasmon polariton (also referred to as surface plasmon) [37]. The frequency of surface plasmons in metal nanoparticles is proportional to the bulk plasma frequency of the metal and strongly depends on the shape, size, and surrounding of the nanoparticle. The interaction (absorption, scattering) 
of light of frequency $\omega$ with a metal nanoparticle is strongest if $\omega$ lies in the vicinity of the particle's surface plasmon frequency. Hence, most methods for optical detection of metal nanoparticles involve excitation sources with frequencies close to the plasmon resonance of the particles under study. For example, the surface plasmon resonance of a small spherical gold particle in water is at about $520 \mathrm{~nm}$, and that of a silver one is at about $400 \mathrm{~nm}$. A metal particle will attenuate a beam of light in which it is placed, partly by absorption, and partly by scattering. The efficiencies of absorption and scattering are characterized by their respective cross-sections, $\sigma_{a b s}$ and $\sigma_{\text {scatt }}$. In the limit of particles small compared to the wavelength, the cross-sections are given by the expressions [25]:

$$
\sigma_{a b s}=-\frac{8 \pi^{2}}{\lambda} R^{3} \operatorname{Im}\left[\frac{m^{2}-1}{m^{2}+2}\right]
$$

and

$$
\sigma_{\text {scatt }}=\frac{128 \pi^{5}}{3 \lambda^{4}} R^{6}\left|\frac{m^{2}-1}{m^{2}+2}\right|^{2},
$$

where $m$ is the ratio of refractive indices of the particle and the medium. In the case of a particle's size being comparable to the wavelength of light, the expressions for $\sigma_{a b s}$ and $\sigma_{\text {scatt }}$ are calculated using the general Mie theory [25] (see Figure 1). In this review, we will mostly focus on detection of $\mathrm{Au}$ and $\mathrm{Ag}$ particles with diameters below $100 \mathrm{~nm}$. Figure 1 shows that for particles of such sizes absorption is larger than scattering. Already for diameters smaller than $50 \mathrm{~nm}$, the limit of "particle small compared to the wavelength" is a very good approximation.

The simplest way of optically detecting metal nanoparticles is via the light they scatter [38]. By tuning the probing wavelength to the plasmon resonance, one considerably improves selectivity against non-metallic objects, and one can access sizes down to a few tens of nanometers $[12,26,27]$. The main drawback of this method is that the scattered intensity steeply decreases for small particles. The scattered field being proportional to the volume of the particle, the intensity decays as $R^{6}$. Despite the large index contrast between the metal and its surroundings, scattering of a single particle smaller than about $30 \mathrm{~nm}$ can no longer be discerned from the scattering of other (often numerous) scatterers. Spatial- or time-resolved enhancement techniques may help, as shown already in 1986 by De Brabander et al., who claimed the detection of single gold particles with a diameter of $5 \mathrm{~nm}$ by video microscopy [39]. However, their integration times of several hundreds of seconds are too long for most applications. For fast optical detection of single metal nanoparticles on a 
scattering background coming from, e.g., organelles in a living cell, one has to find other contrast-enhancing mechanisms.

All of the methods recently proposed for studying single nanoparticles rely on detection of $E_{\text {scatt }}$, the field scattered by the particle, which decreases as $R^{3}$ only. This scattered field is mixed with some suitable, larger, reference field $E_{r e f}$. This requires the reference and scattered waves to be coherent, and their spatial modes to overlap to a large extent (ideally, they should be identical). Otherwise, only the intensities add, and the benefit of the field's weaker size-dependence is lost. Practically, the interference can be implemented in many different ways. The reference wave can be the incident wave itself [40], a reflection of the incident wave on a close-by interface [41-43], the auxiliary wave in a DIC setup [44], in a Michelson interferometer [45], or in a common-path polarization interferometer [36]. The scattered wave can be directly produced by the nanoparticle's dipole itself, or can result from scattering off some other local index inhomogeneity related to the particle. In the photothermal method $[43,44,46]$ this inhomogeneity is induced by heat that the particle releases upon absorption of a pump beam. Note that direct absorption measurements [40] fit the same scheme of mixing a reference and a scattered wave. Indeed, absorption follows from interference of the incident wave with the forward-scattered wave, as stated by the optical theorem [25, 47, 48]. There are different ways to detect small intensity changes resulting from the interference. One can modulate one of the interfering fields, for example by modulating the particle's position [40], or the heating beam [36, 44, 46], and analyze the total intensity with a lock-in amplifier. Alternatively, one can carefully subtract the background by exploiting the frequency- [41], space- or time- dependence of the signal [42, 45]. In practice, all schemes boil down to detecting the interference term, preferably with a tunable phase factor $e^{i \varphi}$ between the two fields:

$$
I=\left|E_{r e f}+e^{i \varphi} E_{s c a t t}\right|^{2}
$$

We will now discuss the signal-to-noise ratio in this general interference experiment. For the sake of simplicity, we assimilate the nanoparticle to a dipole. As discussed above, the full Mie theory introduces significant corrections only for rather large metal particles, so that the correction of the cross-section is typically less than $30 \%$ for diameters under $60 \mathrm{~nm}$. The detected signal arises then only from the interference term $2 \operatorname{Re}\left(E_{r e f}{ }^{*} e^{i \varphi} E_{\text {scatt }}\right)$, whereas the noise is (ideally) limited by the photon noise of the total intensity falling on the detector 
(i.e. mainly the strong reference intensity), and is proportional to $\sqrt{E_{r e f}^{*} E_{r e f}}$. Although different methods may differ in practical details (electronic noise, laser noise, dark counts of the detectors, etc.), for the case of ideal optical and electronic components all of them are ultimately limited by the photon noise only. In these conditions, the signal-to-noise ratio is immediately seen to be independent of the strength of the reference field, at least as long as this field remains much stronger than the scattered field. Therefore, the possibility to detect a scatterer does not depend on the strength of the reference field. In other words, the reference field may be adjusted in each experiment so as to adapt the measured signal to detectors or to other experimental constraints, without any loss (or gain) of signal-to-noise ratio.

We can now give a more quantitative estimate of the maximal signal-to-noise ratio which depends on the physical origin of the signal. Let us first consider the field directly emitted by the induced dipole itself, which, as we recall, is responsible for the extinction of the incident wave, and, for small particles, mainly arises from absorption processes. The number of absorbed photons $N_{a b s}$ during an integration time $T$ is given by the ratio of the absorption cross-section $\sigma_{a b s}$ to the beam area $A$ at the focus. It has to be compared to the number of incident photons $M$ that reach the detector and determine the shot-noise. The signal will be visible when it dominates the shot-noise of $M$, i.e., when

$$
\sigma_{a b s}>\frac{A}{\sqrt{M}} .
$$

The maximum admissible intensity $I_{\text {sat }}$ on the nanoparticle is usually limited by some saturation effect, so that in the best case $M=A I_{\text {sat }} T$. For a single molecule at room temperature, typical values would be $\sigma_{a b s} \approx 0.01 \mathrm{~nm}^{2}, I_{\text {sat }} \approx 1 \mathrm{~kW} \mathrm{~cm}{ }^{-2}$, which would yield a minimum integration time of about $30 \mathrm{~s}$ to directly detect the absorption. This should be compared to the typical integration time in single-molecule fluorescence, often shorter than 1 millisecond. In the same time, for a single gold nanoparticle of $10 \mathrm{~nm}$ in diameter, $\sigma_{a b s} \approx 50 \mathrm{~nm}^{2}$, $I_{\text {sat }} \approx 200 \mathrm{MW} \mathrm{cm}^{-2}$ (approximate intensity to melt the particle in condensed matter). Hence, the minimum integration time for detection is theoretically less than a nanosecond. More practically, the limit for detection by direct absorption [36] or by scattering methods [49] currently seems to lie at diameters of about $5-10 \mathrm{~nm}$ for reasonable integration times of the order of $10 \mathrm{~ms}$.

The case of the photothermal detection largely follows the above discussion but for one 
important difference. The scattered field is now enhanced by the accumulated change of refractive index in a volume $V$ considerably larger than that of the particle. The surrounding medium thus acts as a revelator of the absorbed energy, which explains the great sensitivity of this detection method $[43,44,46]$. The best achieved result was the detection of gold nanoparticles with diameters down to $1.4 \mathrm{~nm}$ with an integration time of $10 \mathrm{~ms}$ and an applied intensity of about $5 \mathrm{MW} \mathrm{cm}^{-2}$ (see Section IV). A full discussion of the origin of the photothermal contrast and of the signal-to-noise ratio has been given by Berciaud et al. [43].

\section{ABSORPTION- AND SCATTERING- BASED METHODS}

In this section we present experiments that directly detect the absorption or scattering of a single metal nanoparticle itself, whereas section IV will focus on the indirect detection of the particle's absorption via scattering by the local environment. As we saw, the sixth-power dependence of the scattered light intensity hinders the detection of very small particles. To circumvent this problem, one rather detects the scattered field, in either of two different ways. One can first directly measure the absorption, which exploits the weaker (third-power) dependence of the absorption cross-section with radius $R$, and amounts to observing the interference of the scattered wave with the incident wave (more precisely, with its imaginary part; the real part has no influence on the absorption). Alternatively, one can measure the interference between the scattered field and a reference field, which again yields a thirdpower size-dependence. The latter method provides more flexibility in experimental design, since both the amplitude and phase of the reference field can be chosen arbitrarily, but one has to ensure a good spatial and temporal coherence between the two waves, while this is done automatically in the first case.

\section{A. Interferometric detection of scattering}

The reference wave for interferometric detection can be created very simply by reflection of the light beam from the surface of the sample's glass substrate, as first proposed by Plakhotnik and Palm [41], and applied by the group of V. Sandoghdar to metal nanoparticles $[49,50]$. The light beam is focused through a microscope objective onto a glass substrate 
on which gold nanoparticles are adsorbed and which is covered by either immersion oil [49] or water [50]. The refractive-index mismatch between the medium and the glass substrate causes a small part of the incident beam to be reflected. This reflected field acts as the reference field $\left(E_{r e f}\right)$ and interferes with the field scattered from the particle $\left(E_{\text {scatt }}\right)$ leading to an intensity at the detector $I_{d e t}$ of

$$
I_{\text {det }}=\left|E_{\text {ref }}+E_{\text {scatt }}\right|^{2}=\left|E_{i}\right|^{2}\left(r^{2}+|s|^{2}-2 r|s| \sin \theta\right)
$$

Here, $r$ is the reflection amplitude of the field, $s=|s| \exp (i \theta)$ is the scattered field amplitude, supposed to be in the same spatial mode as the reference field (e.g., a spherical wave or a Gaussian wave), and $E_{i}$ is the amplitude of the incident field. The sine function arises from the Gouy phase shift, $-\pi / 2$, of the focused reflection. The first of the three terms in equation 5 is a background term, and the second term is the pure scattering contribution, which scales as $R^{6}$ and is dominant for large particles. The third term is the interference term, which scales as $R^{3}$ and is more important than scattering for smaller sizes. Since the pure scattering term and the interference term have opposite signs, the size dependence of the signal has a complex shape, as can be seen in Fig. 2. It is negative for small particles (the cross term dominates) and turns positive for large enough particles (the direct scattering term dominates, as in dark-field detection [27] or total internal reflection [26]). The crossover point depends on the strength of the reference wave, i.e. on the index of refraction of the medium.

The experimental setup described in a recent paper by Jacobsen et al. [50], has two features that are of great interest for biophysical experiments: fast detection and wide-field imaging of single gold nanoparticles. To mimic the fast movement of a nanoparticle, the researchers scan their laser over the particle with a galvo-driven mirror. This allows them to detect single particles of sizes of $\leq 20 \mathrm{~nm}$ in a time window of about $2 \mu \mathrm{s}$. To detect such small particles, however, a laser power of up to $30 \mathrm{~mW}$ (corresponding to an intensity of $30 \mathrm{MW} / \mathrm{cm}^{2}$ ) is needed, which may lead to severe sample heating. Pulsed excitation might help to circumvent this problem.

Since this method does not rely on lock-in amplification, the authors are not confined to confocal detection, but can also do wide-field imaging. This opens a possibility of faster frame rates for single-nanoparticle experiments in biophysics, and integrates better with traditional biophysical experiments. Their paper explains and demonstrates wide-field de- 
tection of single gold particles with diameters down to $15 \mathrm{~nm}$, for the first time with a reasonable integration time. The main noise sources in the wide-field image are laser noise and spatial irregularities on the interface, both leading to spurious features. Jacobsen et al. [50] resorted to numerical post-processing of the images to partially remove these artefacts. Biophysical applications in cells require suppression of the strong scattering background. The authors exploit the particle's plasmon resonance, and subtract two images taken at different wavelengths (532 nm and $488 \mathrm{~nm}$ ), which significantly enhances the nanoparticles' contrast.

A similar scheme for interferometric detection of particle scattering, which is less sensitive to laser intensity noise, has been presented by Ignatovich and Novotny [51]. The experiment (see Fig.3) differs in two important points from that described above: it uses a split photodetector to balance laser noise, and it does not use the sample interface reflection as source for the reference wave. Ignatovich et al. [51] include a Michelson-like interferometer in the beam path, which makes it possible to separately adjust the amplitude of the reference field and the scattered field from the particle, while keeping the reference field larger than the backscattered field from the sample background. Off-center scatterers produce a signal, while the static background (the $r^{2}$ term in equation 5 ) is the same for both detector halves and consequently drops out. This renders the method essentially background-free, as long as the beam pointing stability of the laser source is high enough. Applying this method to a clean flowing liquid solution, Ignatovich and Novotny could detect single gold nanoparticles with sizes down to $14 \mathrm{~nm}$, polystyrene beads with sizes down to $30 \mathrm{~nm}$, and unlabelled single viruses with a detection bandwidth of $10 \mathrm{kHz}$ [51].

\section{B. Direct detection of absorption and transient absorption}

A different approach is to directly detect the absorption of incident light by a single particle. A gold nanoparticle with a diameter of $5 \mathrm{~nm}$ has an absorption cross-section of about $5 \mathrm{~nm}^{2}$ at the plasmon resonance. This cross-section is about 5 orders of magnitude smaller than the focal spot size of a good microscope objective, i.e. the presence of the particle reduces the detected intensity by $10^{-5}$. Such small variations in the transmitted light intensity can be detected if they are larger than the laser intensity fluctuations and the always-present photon noise. Arbouet et al. [40] chose to spatially modulate the position of 
the nanoparticle in the focus of the laser beam with a fast piezo translator, while illuminating the particle by a continuous laser beam of wavelength $\lambda=532 \mathrm{~nm}$. In this way, they are able to extract the small variation of the transmitted power with a lock-in amplifier and report detection of single gold particles as small as $5 \mathrm{~nm}$ in diameter.

Careful calibration of the detection path allows the authors to make absolute measurements of the absorption cross-sections. Knowing this value, one can address the following common problem of single metal particle experiments. In general, the particles are spincoated on a glass substrate to be observed in a microscope. The dielectric surrounding of the particles is therefore highly asymmetric, glass on one side and air on the other side. This makes it difficult to choose a value for the dielectric constant of the medium, $\epsilon_{d}=n_{d}^{2}$, which enters the calculation of the cross sections (equations 1,2 ) via $m=n_{\text {metal }} / n_{d}$. Arbouet $e t$ al. [40] show experimentally that it suffices to use the average of the dielectric constants of air and glass for $\epsilon_{d}$ (Fig. 4). Through the absolute value of the cross-section, it is also possible (knowing $\epsilon_{d}$ ) to accurately determine the size of the particle [52].

Instead of the average cw absorption of a metal nanoparticle, the time-dependent absorption can be measured in pump-probe experiments. Let us first summarize the relaxation chain following absorption of an ultra-short light pulse. Just after the pulse, some of the particle's free electrons have been excited. These excited electrons quickly thermalize with the other electrons, forming a hot gas which subsequently cools by heating the lattice via emission of phonons. By varying the delay between the pump and probe pulses, one obtains information on the electron-phonon coupling times. A thermodynamic model of two coupled differential equations, the two-temperature model [53], is generally used to describe the interactions of the electron gas and the lattice. In addition to fast electronic relaxation, the sudden heating of the particle's lattice and the electron pressure of the hot electron gas trigger mechanical oscillations of the metal nanoparticle, which have already been observed in ensemble measurements $[15,16]$. Because they modify the size or shape of the particle, these elastic oscillations couple to the optical response, mainly via a shift of the surface plasmon resonance.

Van Dijk et al. [36] have recently performed pump-probe experiments on single gold nanoparticles with a common-path interferometer. The interferometer is based on time and polarization division, rather that on spatial division, of probe and reference beams. Before entering the microscope, a 1-ps laser pulse is split into two pulses by a thick birefringent 
crystal with its fast axis oriented at 45 degrees with respect to the polarization of the probe. This yields two pulses, a probe and a reference pulse, which are orthogonally polarized and have a mutual delay of 10 ps. Behind the microscope, the two pulses are recombined again. The interference of the two waves provides information on the change in permittivity of the sample in the 10-ps interval between the pump and the probe pulses. This change is triggered by the near-infrared pump pulse. By varying the delay between the pump and the probe-reference pair, ultrafast changes in optical properties of a single particle can be studied. The interferometer is combined with intensity modulation of the pump at $400 \mathrm{kHz}$ and lock-in detection. With this method Van Dijk et al. were able to analyze the acoustic vibrations of single gold nanoparticles as small as $50 \mathrm{~nm}$, as well as the stronger and faster electronic response for particles with diameters down to $10 \mathrm{~nm}$.

Figure 5 illustrates the advantage of single-particle measurements of acoustic vibrations over ensemble studies, which had been measured previously in several laboratories $[16,54,55]$. In this experiment, vibrations of 29 individual particles were measured under the same conditions. The average of the 29 traces that were obtained approximates the ensemble response. Due to inhomogeneous broadening, the ensemble's oscillation appears to damp much faster that those of individual particles. The damping of the individual particles is the pure homogeneous damping of the vibration, due to mechanical coupling with the environment. The elastic properties of the environment and of the contact area determine the damping of the vibration and can be probed in this way. Asymmetric coupling of the particle to the environment is thought to launch acoustical oscillations with broken spherical symmetry [36], which are not accessible in ensemble pump-probe spectroscopy. Examples of such time-traces and of their Fourier transforms are shown in Fig. 6. In addition to the main breathing mode, the additional quadrupolar mode appears at a lower frequency. These acoustical oscillations make nanoparticles interesting mechanical probes for their environment, which can be addressed in the optical far-field.

The more classical pump-probe method was applied in a recent paper by Muskens et al. [35], who obtain information on the electron-phonon coupling times on a particle-by-particle basis. In their configuration, a single silver nanoparticle is excited by femtosecond pulses of a Ti:sapphire laser (800-900 nm). Its frequency-doubled output, which can be tuned between 400 and $450 \mathrm{~nm}$, is used to probe the absorption as a function of the pump-probe time delay. Amplitude-modulation of the pump beam is used for lock-in detection. The 
measured electron-phonon coupling times are in good agreement with the two-temperature model, confirming earlier ensemble experiments.

\section{PHOTOTHERMAL DETECTION}

This section presents a detailed description of photothermal methods developed for the detection of minute absorbing nanoobjects. Excited near their plasmon resonance, metal nanoparticles have a large absorption cross-section and exhibit a fast electron-phonon relaxation time in the picosecond range [56], which makes them very efficient light absorbers. The luminescence yield of these particles being extremely weak [19, 57], almost all the absorbed energy is converted into heat. The increase of temperature induced by this absorption gives rise to a local variation of the refractive index. This photothermal effect can be used to detect and study the nanoparticles.

Photothermal detection was proposed earlier by Tokeshi et al. [58], who used a thermal lens effect to detect very low concentrations of absorbing molecules in liquid solutions. Boyer et al. [44] designed a very sensitive polarization interference method, called Photothermal Interference Contrast (PIC), for the detection of the small refractive index changes around an absorbing particle. The experiment was performed with two lasers. The horizontally polarized beam of a He-Ne laser (633 nm wavelength) was split into two beams (probe and reference beams) by a Wollaston prism and sent to the microscope objective through a telecentric lens system. The beams back-reflected by the sample, having the same optical path as the incident ones, recombined in the Wollaston prism. The vertically polarized recombination reflected by a polarizing cube beamsplitter was sent to a fast photodiode. The nanoparticles were heated by the 514-nm beam of an argon laser, whose intensity was modulated at high frequency (few hundreds of $\mathrm{kHz}$ ) by an acousto-optical modulator. A lockin amplifier detected the variations of the red intensity and thus the phase difference between the two red beams at the modulation frequency of the green beam with an integration time of $10 \mathrm{~ms}$. Microscopic images were obtained by scanning the sample with respect to the three spots. With PIC, images of gold nanoparticles down to $5 \mathrm{~nm}$ in diameter embedded in thin polymer films were recorded with a signal-to-noise ratio larger than $\sim 10$. The authors also showed that, in addition to their intrinsic sensitivity, the photothermal methods are remarkably insensitive to scattering background, even when arising from such 
strong scatterers as 300-nm latex beads. Using a modified PIC setup in order to image nanoparticles in 3D environments, Cognet et al. [59] could detect single proteins labelled with individual 10-nm gold nanoparticles at the surface of fixed cells. The sensitivity of the PIC method, although high, did not reach the shot noise limit. Indeed, the use of high numerical-aperture objectives induced depolarization effects which degraded the quality of the overlap between the two arms of the interferometer. As a consequence, PIC required high laser intensities $\left(\approx \mathrm{MW} / \mathrm{cm}^{2}\right)$, which for biological applications can be a serious limitation.

Berciaud et al. recently developed a more sensitive photothermal method, called Photothermal Heterodyne Imaging (PHI)[46], which does not suffer from the previous limitations. It combines a time-modulated heating beam and a non-resonant probe beam, overlapping on the sample. The probe beam produces a frequency-shifted scattered field as it interacts with the time-modulated variations of the refractive index around the absorbing nanoparticle. The scattered field is then detected through its beatnote with the probe field which plays the role of a local oscillator as in any heterodyne technique. This signal is extracted by lock-in detection. In practice, the sensitivity of PHI is two orders of magnitude higher than that of other methods and is shot-noise limited. Particles with $5 \mathrm{~nm}$ diameter are detected with a signal to noise ratio of about 30 at excitation intensities of $400 \mathrm{~kW} / \mathrm{cm}^{2}$ and with an integration time of $5 \mathrm{~ms}$. This sensitivity enabled the unprecedented detection of individual gold nanoparticles as small as $1.4 \mathrm{~nm}$ in diameter, with only 67 gold atoms (see Figure 7). In addition, since the PHI signal is directly proportional to the power absorbed by the nano-object, this method has been used to perform the first absorption spectroscopy study of individual gold nanoparticles down to diameters of $5 \mathrm{~nm}$ [34] as will be discussed below.

Several theoretical models predict the existence of so-called intrinsic size effects in the optical response of metallic nanoparticles with sizes significantly smaller than the electron mean free path $[1,18]$. Limitations of the electron mean free path as well as damping by the chemical interface $[1,60]$ increase the damping rate of the surface plasmon resonance, leading to shorter dephasing times (down to a few fs). Due to these intrinsic size effects, the dielectric permittivity of a nanoparticle differs from that of the bulk metal because of the additional surface damping contributions. Experimental studies on ensembles of metallic nanoparticles revealed the existence of such effects $[1,61]$. However, a quantitative description of these effects was difficult for ensembles, mostly because of inhomogeneous broadening. In order 
to circumvent this shortcoming, one can use PHI to record absorption spectra of individual gold nanoparticles with diameters down to $5 \mathrm{~nm}$ with excellent signal-to-noise ratio [34]. Figure 8 represents the absorption spectra of 2 single gold nanoparticles with diameters of $33 \mathrm{~nm}$ and $5 \mathrm{~nm}$ respectively, performed in the $515-580 \mathrm{~nm}$ wavelength range. The values of peak resonance energies are not particularly affected by intrinsic size effects. In contrast, a significant increase in width of the resonance clearly appears, which cannot be described by Mie's theory with the bulk values of the gold dielectric constant [62]. A good agreement was found between the experimental widths and Mie simulations with size-dependent corrections [34] (Figure 8(b)).

Although the existence of intrinsic size effects in the optical response of gold nanoparticles was unambiguously revealed, part of the damping processes are due to interband transitions, which makes it difficult to connect the widths of the plasmon resonances to the damping rate. Consequently, the plasmon spectra of individual gold particles are asymmetric, and it is delicate (even impossible for very small particles) to define a full-width-at-half maximum of absorption spectra. To suppress this additional damping channel, one could red-shift the plasmon resonance frequency beneath the onset of interband transitions, either by embedding gold nanospheres in a high-index matrix [63], or by studying the long-axis plasmon mode of gold nanorods [64], or by using core-shell nanoparticles [65]. Another possibility is to use silver nanospheres [27], since their resonant energies are well separated from interband transitions [62]. A drawback of silver particles is their weak photostability due to photooxidation [30], but the reactivity of silver particles can be reduced by encapsulation, e.g., with PEG (poly(ethylene glycol))[66, 67]. Berciaud et al. [43] demonstrated the detection of individual PEG-coated silver nanoparticles with an average diameter of $5.3 \mathrm{~nm}$ with the PHI method. The size distribution, however, was broad. In this case they used a modulated diode laser emitting at $405 \mathrm{~nm}$ as heating source with an intensity of $\sim 50 \mathrm{~kW} / \mathrm{cm}^{2}$. As expected, the signal obtained with silver particles of $5.3 \mathrm{~nm}$ in diameter is about 10 times higher than that of gold particles of the same size, when identical heating intensities are used to excite the nanoparticles at the peak of their surface plasmon resonance. Expected progress in the synthesis of silver nanoparticles with narrower size distributions should permit a quantitative study of the surface plasmon resonance of individual silver particles much smaller than $5 \mathrm{~nm}$ in diameter.

Interestingly, PHI can also be used to detect $\mathrm{CdSe} / \mathrm{ZnS}$ semiconductor nanocrystals. 
Those nano-objects have relatively high absorption cross-sections [68, 69] (typically few $10^{-} 16 \mathrm{~cm}^{2}$ ) and when excited with sufficiently high intensities, excitons are created at average rates significantly higher than their radiative recombination rates $[69,70]$. In this regime, efficient non-radiative Auger recombination of the prepared multi-excitons takes place [71]. In CdSe/ZnS nanocrystals, these recombination processes occur in the picosecond time range, as measured in time-resolved experiments on ensemble samples [72]. Individual nanocrystals could thus be detected through their absorption using the PHI method [46] and photothermal absorption spectra could be recorded in the high $\mathrm{cw}$ excitation regime, where excitons are created at a high rate. Comparison with ensemble measurements gave estimates of the biexciton and trion binding energies, which are in good agreement with prior theoretical and experimental results [73].

The PHI method is very promising for applications in biosciences. In a first demonstration, Blab et al. [74] showed the applicability of PHI for a new readout strategy of DNA micro-arrays based on gold nanoparticles. PHI enables direct counting of individual nanoparticles on each array spot and stable signals, without any silver enhancement. Given the possibility to detect particles down to nanometer sizes (for which steric hindrance is minimal), the linear dynamic range of the method is particularly large and well suited for micro-array detection. For live-cell imaging, and unlike other methods, PHI allows for the first time the detection of very small metal nanoparticles $(<5 \mathrm{~nm})$ in highly scattering environments with intensities compatible with cell integrity. For such applications, however, the backward configuration described above cannot be used, as nanoparticles do not always lie at the coverslip/sample interface. For such thick samples, one can use the forward-scattered field by introducing a second microscope objective $(80 \mathrm{x}, \mathrm{NA}=0.8)$ which efficiently collects the interfering probe-transmitted and forward-scattered fields [43]. An image of live neurons with 5-nm-gold-labelled glutamate receptors on their outer membrane is presented as an example in Figure 9. Precise 3D imaging of individual $5 \mathrm{~nm}$ nanoparticles embedded in an agarose gel was possible, and particles as far as $10 \mu \mathrm{m}$ from the surface could be imaged. As the PHI method requires a raster scan of the sample with typically a few-millisecond integration time per point, fast imaging rates can not be readily obtained. Lasne et al. [75] recently designed a tracking scheme based on a triangulation from three measurement points to record the trajectories of single membrane proteins labelled with gold nanoparticles in live cells at video rate. Single metal nanoparticle tracking combines the advantages of small 
marker size (comparable to the sizes of single fluorescent dyes) with practically unlimited observation times owing to the high chemical stability of gold particles.

\section{CONCLUSIONS}

The present review has given a partial survey of a young field, the optical study of individual metallic nanoparticles. Among the many methods available in far-field optical microscopy, we chose to restrict our discussion to techniques in which the particle responds at the same wavelength as the probing laser. A number of new detection schemes have recently flourished. They all boil down to interference of the particle's scattered wave with a suitable reference wave. Since the interference term decreases as the particle's volume only (instead of its square, as for the scattered intensity in dark field), these methods are well-suited to the detection of small particles. In order to study the properties of the particle itself, it is convenient to detect the wave directly scattered by particle. This is particularly important to access its response at picoseconds and shorter times. For the purposes of detection and tracking, particularly in biological applications, sensitivity is central. Photothermal methods enhance the scattered wave through an accumulated change of the index of refraction in a volume larger than that of the particle itself. The heating is caused by the particle's absorbing an auxiliary beam. The surrounding medium thus acts as a specific revelator of absorption processes, which leads to excellent selectivity against background from nonabsorbing scatterers. As it has been recently demonstrated, this is particularly attractive for the study of single gold-labelled molecules in live cells. With this broad assortment of powerful new methods, we may soon expect many fascinating applications in cellular biology and nanoscience.

Acknowledgements : The authors gratefully acknowledge the Foundation for Fundamental Research on Matter (FOM), and the European Commission (IST contract 200133578) for the financial support. A. T. acknowledges the financial support from the Dutch Organisation for Scientific Research (NWO). The Bordeaux Nanophotonics group acknowledges C.N.R.S., the French Ministry for Education and Research (ACI Nanoscience and DRAB), and the Région Aquitaine for financial support. 
[1] U. Kreibig and M. Vollmer, Optical Properties of Metal Clusters, vol. 25 of Springer Series in Materials Science (Springer, Berlin, 1995).

[2] M. Salerno, J. R. Krenn, B. Lamprecht, G. Schider, H. Ditlbacher, N. Felidj, A. Leitner, and F. Aussenegg, Opto-Electron. Rev. 10, 217 (2002).

[3] J. R. Krenn, Nat. Mater. 2, 210 (2003).

[4] S. Maier, P. G. Kik, H. A. Atwater, S. Meltzer, E. Harel, B. Koel, and A. A. G. Requicha, Nat. Mater. 2, 229 (2003).

[5] W. L. Barnes, A. Dereux, and T. W. Ebbesen, Nature 424, 824 (2003).

[6] Y. H. Liau, A. N. Unterreiner, Q. Chang, and N. F. Scherer, J. Phys. Chem. B 105, 2135 (2001).

[7] D. Yelin, D. Oron, S. Thiberge, E. Moses, and Y. Silberberg, Opt. Express 11, 1385 (2003).

[8] J. Nappa, G. Revillod, I. Russier-Antoine, E. Benichou, C. Jonin, and P. F. Brevet, Phys. Rev. B 71, 165407 (2005).

[9] H. Ditlbacher, J. R. Krenn, B. Lamprecht, A. Leitner, and F. R. Aussenegg, Opt. Lett. 25, $563(2000)$.

[10] K. Kneipp, H. E. H. Kneipp, I. Itzkan, R. R. Dasari, and M. Feld, J. Phys.: Condens. Matter 14, R597 (2002).

[11] H.-G. Boyenand, G. Kästle, F. Weigl, B. Koslowski, C. Dietrich, P. Ziemann, J. Spatz, S. Riethmüller, C. Hartmann, M. Möller, et al., Science 297, 1533 (2002).

[12] J. Yguerabide and E. E. Yguerabide, Anal. Biochem. 262, 157 (1998).

[13] G. Raschke, S. Kowarik, T. Franzl, C. Sönnichsen, T. A. Klar, J. Feldmann, A. Nichtl, and K. Kurzinger, Nano Lett. 3, 935 (2003).

[14] S. Link and M. A. El-Sayed, J. Phys. Chem. B 103, 4212 (1999).

[15] C. Voisin et al., J. Phys. Chem. B 105, 2264 (2001).

[16] G. V. Hartland, Phys. Chem. Chem. Phys. 6, 5263 (2004).

[17] B. Lamprecht, J. R. Krenn, A. Leitner, and F. R. Aussenegg, Phys. Rev. Lett. 83, 4421 (1999).

[18] S. Link and M. A. El-Sayed, Int. Rev. Phys. Chem. 19, 409 (2000).

[19] E. Dulkeith, T. Niedereichholz, T. A. Klar, J. Feldmann, G. von Plessen, D. I. Gittins, K. S. Mayya, and F. Caruso, Phys. Rev. B 70, 205424 (2004). 
[20] B. Palpant, H. Portales, L. Saviot, J. Lermé, B. Prével, M. Pellarin, E. Duval, A. Perez, and M. Broyer, Phys. Rev. B 60, 17107 (1999).

[21] A. Nelet, A. Crut, A. Arbouet, N. Del Fatti, F. Vallée, H. Portales, L. Saviot, and E. Duval, Appl. Surf. Sci. 226, 209 (2004).

[22] R. Hillenbrand and F. Keilmann, Appl. Phys. B 73, 239 (2001).

[23] G. P. Wiederrecht, Eur. Phys. J.-Appl. Phys. 28, 3 (2004).

[24] G. Mie, Ann. Physik 25, 377 (1908).

[25] H. C. van de Hulst, Light Scattering by Small Particles (Dover Publications, 1981).

[26] C. Sönnichsen, S. Geier, N. E. Hecker, G. von Plessen, J. Feldmann, H. Ditlbacher, B. Lamprecht, J. R. Krenn, F. R. Aussenegg, V. Z. H. Chan, et al., Appl. Phys. Lett. 77, 2949 (2000).

[27] S. Schultz, D. R. Smith, J. J. Mock, and D. A. Schultz, Proc. Natl. Acad. Sci. U. S. A. 97, $996(2000)$.

[28] M. R. Beversluis, A. Bouhelier, and L. Novotny, Phys. Rev. B 68, 115433 (2003).

[29] C. D. Geddes, A. Parfenov, and J. R. Lakowicz, J. Fluoresc. 13, 297 (2003).

[30] L. A. Peyser, A. E. Vinson, A. P. Bartko, and R. M. Dickson, Science 291, 103 (2001).

[31] M. Lippitz, M. A. van Dijk, and M. Orrit, Nano Lett. 5, 799 (2005).

[32] M. Pelton, M. Liu, S. Park, and N. F. Scherer, ArXiv:cond-mat/ v4, 0506158 (2005).

[33] M. A. van Dijk, M. Lippitz, and M. Orrit, Acc. Chem. Res. 38, 594 (2005).

[34] S. Berciaud, L. Cognet, P. Tamarat, and B. Lounis, Nano Lett. 5, 515 (2005).

[35] O. L. Muskens, N. Del Fatti, and F. Vallée, Nano Lett. 6, 552 (2006).

[36] M. A. van Dijk, M. Lippitz, and M. Orrit, Phys. Rev. Lett. 95, 267406 (2005).

[37] H. Raether, Surface Plasmons, vol. 111 of Springer Tracts in Modern Physics (Springer, Berlin, 1988).

[38] J. Gelles, B. J. Schnapp, and M. P. Sheetz, Nature 331, 450 (1988).

[39] M. Debrabander, R. Nuydens, G. Geuens, M. Moeremans, and J. Demey, Cell Motil. Cytoskeleton 6, 238 (1986).

[40] A. Arbouet, D. Christofilos, N. Del Fatti, F. Vallée, J. R. Huntzinger, L. Arnaud, P. Billaud, and M. Broyer, Phys. Rev. Lett. 93, 127401 (2004).

[41] T. Plakhotnik and V. Palm, Phys. Rev. Lett. 87, 183602 (2001).

[42] K. Lindfors, T. Kalkbrenner, P. Stoller, and V. Sandoghdar, Phys. Rev. Lett. 93, 037401 
(2004).

[43] S. Berciaud, D. Lasne, G. A. Blab, L. Cognet, and B. Lounis, Phys. Rev. B 73, 045424 (2006).

[44] D. Boyer, P. Tamarat, A. Maali, B. Lounis, and M. Orrit, Science 297, 1160 (2002).

[45] F. V. Ignatovich and L. Novotny, Phys. Rev. Lett. 96, 013901 (2006).

[46] S. Berciaud, L. .Cognet, G. A. Blab, and B. Lounis, Phys. Rev. Lett. 93, 257402 (2004).

[47] H. C. van de Hulst, Physica 15, 740 (1949).

[48] M. Born and E. Wolf, Principles of Optics $6^{\text {th }}$ ed. (Pergamon Press, Oxford, England, 1986).

[49] K. Lindfors et al., Phys. Rev. Lett. 93, 037401 (2004).

[50] V. Jacobsen, P. Stoller, C. Brunner, V. Vogel, and V. Sandoghdar, Opt. Express 14, 405 (2006).

[51] F. V. Ignatovich and L. Novotny, Phys. Rev. Lett. 96, 013901 (2006).

[52] O. L. Muskens, N. Del Fatti, F. Vallée, J. R. Huntzinger, P. Billaud, and M. Broyer, Appl. Phys. Lett. 88, 063109 (2006).

[53] S. D. Brorson, A. Kazeroonian, J. S. Moodera, D. W. Face, T. K. Cheng, E. P. Ippen, M. S. Dresselhaus, and G. Dresselhaus, Phys. Rev. Lett. 64, 2172 (1990).

[54] N. Del Fatti, C. Voisin, M. Achermann, S. Tzortzakis, D. Christofilos, and F. Vallée, Phys. Rev. B 61, 16956 (2000).

[55] M. Perner, S. Grésillon, J. Marz, G. von Plessen, J. Feldmann, J. Porstendorfer, K. J. Berg, and G. Berg, Phys. Rev. Lett. 85, 792 (2000).

[56] S. Link and M. A. El-Sayed, Annual Rev. Phys. Chem. 54, 331 (2003).

[57] J. P. Wilcoxon, J. E. Martin, F. Parsapour, B. Wiedenman, and D. F. Kelley, J. Chem. Phys. 108, 9137 (1998).

[58] M. Tokeshi, M. Uchida, A. Hibara, T. Sawada, and T. Kitamori, Anal. Chem. 73, 2112 (2001).

[59] L. Cognet, C. Tardin, D. Boyer, D. Choquet, P. Tamarat, and B. Lounis, Proc. Natl. Acad. Sci. U. S. A. 100, 11350 (2003).

[60] B. N. J. Persson, Surf. Sci. 281, 153 (1993).

[61] J. Bosbach, C. Hendrich, F. Stietz, T. Vartanyan, and F. Träger, Phys. Rev. Lett. 89, 257404 (2002).

[62] P. B. Johnson and R. W. Christy, Phys. Rev. B 6, 4370 (1972).

[63] T. Klar, M. Perner, S. Grosse, G. von Plessen, W. Spirkl, and J. Feldmann, Phys. Rev. Lett. 80, 4249 (1998). 
[64] C. Sönnichsen, T. Franzl, T. Wilk, G. von Plessen, J. Feldmann, O. Wilson, and P. Mulvaney, Phys. Rev. Lett. 88, 077402 (2002).

[65] E. Prodan, C. Radloff, N. J. Halas, and P. Nordlander, Science 302, 419 (2003).

[66] J. Zheng and R. M. Dickson, J. Am. Chem. Soc. 124, 13982 (2002).

[67] W. P. Wuelfing, S. M. Cross, D. T. Miles, and R. W. Murray, J. Am. Chem. Soc. 120, 12696 (1998).

[68] C. A. Leatherdale, W. K. Woo, F. V. Mikulec, and M. G. Bawendi, J. Phys. Chem. B 106, 7619 (2002).

[69] B. Lounis, H. A. Bechtel, D. Gerion, P. Alivisatos, and W. E. Moerner, Chem. Phys. Lett. 329, 399 (2000).

[70] G. Schlegel, J. Bohnenberger, I. Potapova, and A. Mews, Phys. Rev. Lett. 88, 137401 (2002).

[71] A. L. Efros, D. J. Lockwood, and L. Tsybeskov, eds., Semiconductor Nanocrystals. Nanostructure Science and Technology. (Kluwer Academic / Plenum Publishers, New York, 2003).

[72] V. I. Klimov, A. A. Mikhailovsky, D. W. McBranch, C. A. Leatherdale, and M. G. Bawendi, Science 287, 1011 (2000).

[73] S. Berciaud, L. Cognet, and B. Lounis, Nano Lett. 5, 2160 (2005).

[74] G. A. Blab, L. Cognet, S. Berciaud, I. Alexandre, D. Husar, J. Remacle, and B. Lounis, Biophys. J. 90, L13 (2006).

[75] D. Lasne, G. A. Blab, S. Berciaud, M. Heine, L. Groc, D. Choquet, L. Cognet, and B. Lounis, submitted (2006).

[76] H. Lamb, Proceedings of the London Mathematical Society 13, 189 (1882). 
Fig. 1 Absorption and scattering cross-sections of gold nanospheres in water as a function of diameter, calculated with Mie theory.

Fig. 2 Size dependence of the change in detected signal, normalized to the background signal, for the detection scheme of Jacobsen et al. [50]. The data for two different wavelengths (532 nm, filled squares; $488 \mathrm{~nm}$, open circles) show the resonant origin of the signal. The error bars in particle size are due to the size distribution in the sample. The error in intensity gives the spread over about 10 particles of each size.

Fig. 3 Interferometric detection of scattering gold nanoparticles in a flow channel as demonstrated by Ignatovich et al. [51]. (a) The scattered light field is interferometrically mixed with a reference field and detected on a split photodiode. The normalized difference of the detector halves gives a background-free signal. When a single nanoparticle flows through a narrow channel (shown in part (b)), it produces a peak as shown in (c) with a width of a few milliseconds.

Fig. 4 The detection scheme of Arbouet et al. [40] allows the authors the absolute measurement of the gold nanoparticle's absorption cross-section (right axis). This can be compared to the prediction by Rayleigh scattering theory, using different values for the environment permittivity $\epsilon_{d}$. The particles are prepared on a glassair interface. A pure glass or air environment does not describe the data (air, dotted, $\epsilon_{d}=1$; glass, dash-dotted, $\left.\epsilon_{d}=2.25\right)$. The data can be described by a mean permittivity $\left(\epsilon_{d}=1.6\right)$, with (full line) or without (dashed line) taking into account the increased electron damping due to surface scattering for smaller particles. The inset shows a histogram of transmission changes $\delta T / T$ for one particle size. It demonstrates that indeed single particles and not aggregates are detected.

Fig. 5 Comparison of delay scans (top panel) and power spectra of the oscillatory part (bottom panel) of a set of 29 single gold nanoparticles (examples shown as thin black lines) to a reconstructed ensemble measurement (thick grey line). For this experiment the sample was covered by index-matching fluid, which increased the damping of oscillations. The particles have a nominal size of $50 \mathrm{~nm}$ with a spread 
of $6 \%$. The average spectrum in part (B) is scaled by 42 for clarity. The quality factor $Q=\nu / \Delta \nu$ is 4 for the ensemble and about 13 for the individual traces.

Fig. 6 (A) Delay scan of a single gold nanoparticle. The oscillation pattern shows a complex modulation. (B) Power spectra of this particle's oscillation (top spectrum) and of two other particles. Frequencies and amplitudes are normalized to those of the $(0,0)$ mode (The absolute frequencies were $67 \mathrm{GHz}, 59 \mathrm{GHz}, 63 \mathrm{GHz}$, from top to bottom). The power spectra clearly show, besides the fundamental breathing mode, an additional mode at the same relative frequency to the breathing mode, for each of the three particles. This mode is ascribed to a shear deformation of the particle. The low frequency peak (top spectrum: $28 \mathrm{GHz}$ ) lies between frequencies of the $(0,2)$ mode for free boundary (F, thick line on bar) and for rigid boundary ( $R$, end of bar), calculated according to Lamb's theory of a vibrating elastic sphere [76].

Fig. 7 Top: 3D representation of a photothermal heterodyne image $\left(5 \times 5 \mu \mathrm{m}^{2}\right)$ containing individual gold-clusters of $1.4 \mathrm{~nm}$ diameter. The particles are detected with a relatively small heating power $(\sim 3.5 \mathrm{~mW})$ and a remarkably large signal-to-noise ratio (larger than 10) at an integration time of $10 \mathrm{~ms}$. Bottom: Size dependence of the absorption cross-section of gold nanoparticles with diameters ranging from $1.4 \mathrm{~nm}$ to $75 \mathrm{~nm}$. Samples containing nanoparticles of two different (successive) sizes $(1.4 \& 5 \mathrm{~nm}, 2 \& 5 \mathrm{~nm}, 5 \& 10 \mathrm{~nm}$ up to $33 \& 75 \mathrm{~nm})$ are prepared. For each sample, a histogram of the signal amplitudes was generated as shown in (a). All the histograms displayed bimodal distributions and the mean of each population was measured. This allows one to calibrate the size dependence of the absorption cross-section normalized to that of $10 \mathrm{~nm}$ particles (b). A good qualitative agreement with a third-order law of the absorption cross-section vs the radius of the particles is obtained (solid line in (b)) as expected by the Mie scattering theory.

Fig. 8 Left: Normalized absorption spectra of 2 single gold nanoparticles of diameters $33 \mathrm{~nm}$ (gray open circles) and $5 \mathrm{~nm}$ (black open squares). The extracted width at half-maximum is shown on both nanoparticle spectra. The experimental spectra 
are compared with simulations based on Mie theory (solid lines) using a sizedependent modification in the dielectric constant of gold. Right: Size-dependence of the plasmon resonance width. Experimental data (circles with standard deviations) are compared with Mie theory without (dotted line) and with (gray area) size-dependent correction. The gray area accounts for the experimental uncertainties on the bulk dielectric function of gold given in [62].

Fig. 9 Superimposed bright field and PHI images of a $20 \mu \mathrm{m} \times 20 \mu \mathrm{m}$ portion of live cultured hippocampal neurons with GluR2 receptors labelled with $5 \mathrm{~nm}$ gold nanoparticles. The PHI image (black spots and stripes) reveal the presence of point-like signals which correspond to immobile GluR2-linked nanoparticles. Most nanoparticles move during the raster scan of the sample, which produces characteristic stripe signals. The excitation intensity is $400 \mathrm{~kW} / \mathrm{cm}^{2}$, the integration time $5 \mathrm{~ms}$ and one pixel is $150 \mathrm{~nm}$ in size. 


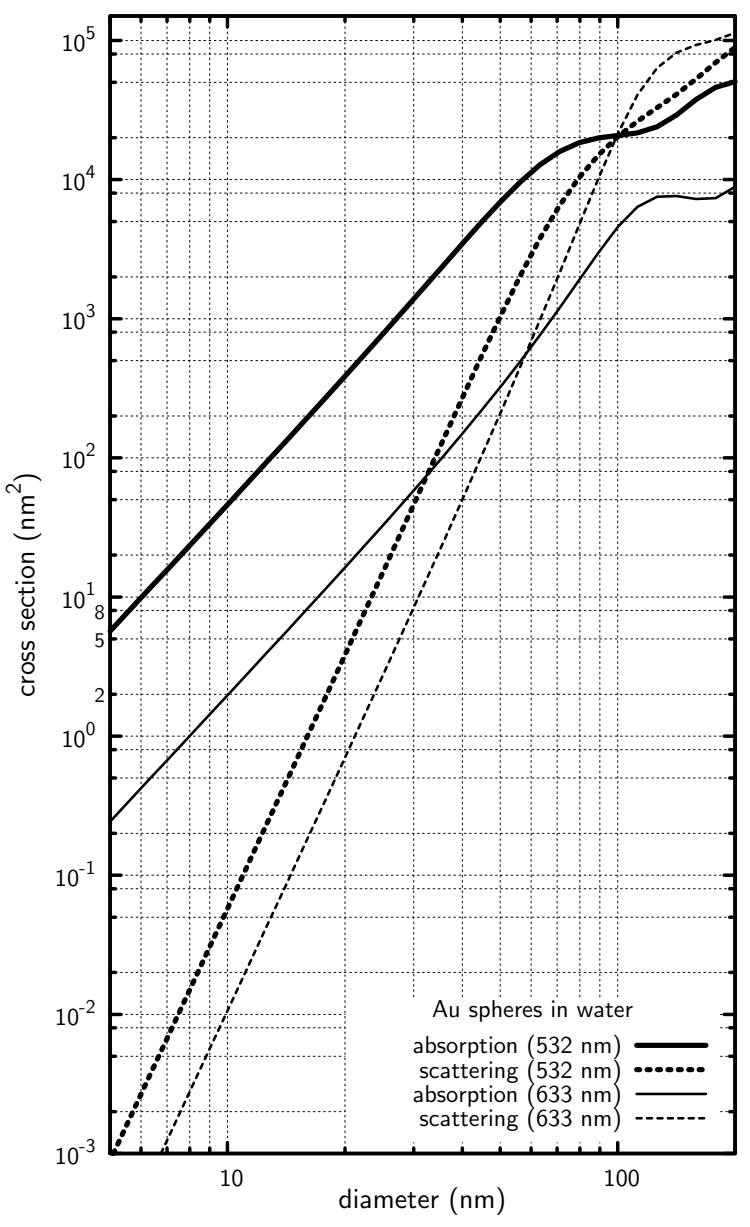

FIG. 1: Absorption and scattering cross-sections of gold nanospheres in water as a function of diameter, calculated with Mie theory. 


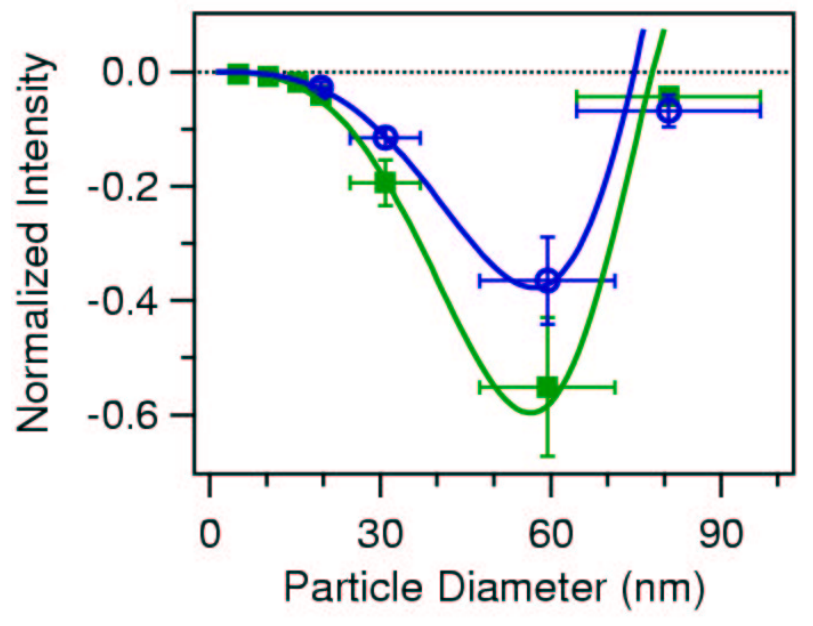

FIG. 2: Size dependence of the change in detected signal, normalized to the background signal, for the detection scheme of Jacobsen et al. [50]. The data for two different wavelengths (532 nm, filled squares; $488 \mathrm{~nm}$, open circles) show the resonant origin of the signal. The error bars in particle size are due to the size distribution in the sample. The error in intensity gives the spread over about 10 particles of each size. 


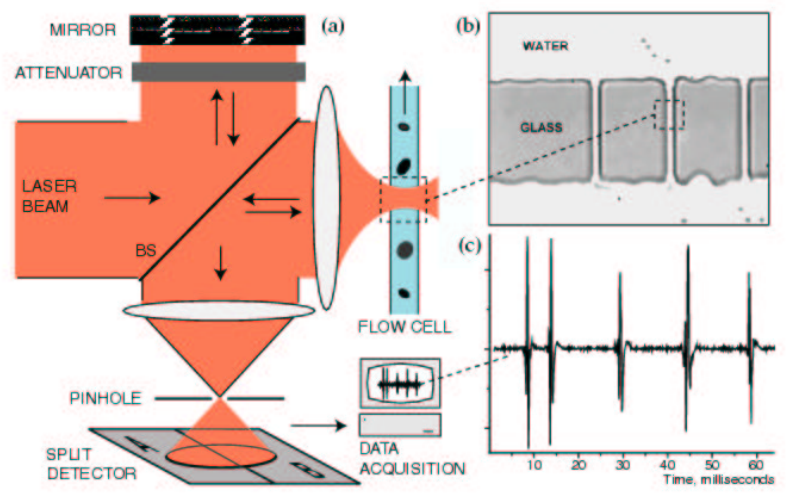

FIG. 3: Interferometric detection of scattering gold nanoparticles in a flow channel as demonstrated by Ignatovich et al. [51]. (a) The scattered light field is interferometrically mixed with a reference field and detected on a split photodiode. The normalized difference of the detector halves gives a background-free signal. When a single nanoparticle flows through a narrow channel (shown in part (b)), it produces a peak as shown in (c) with a width of a few milliseconds. 


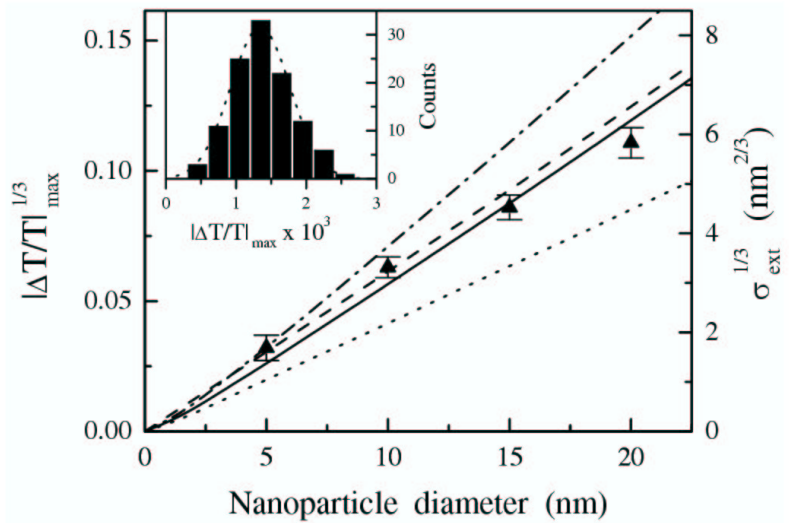

FIG. 4: The detection scheme of Arbouet et al. [40] allows the authors the absolute measurement of the gold nanoparticle's absorption cross-section (right axis). This can be compared to the prediction by Rayleigh scattering theory, using different values for the environment permittivity $\epsilon_{d}$. The particles are prepared on a glass-air interface. A pure glass or air environment does not describe the data (air, dotted, $\epsilon_{d}=1$; glass, dash-dotted, $\epsilon_{d}=2.25$ ). The data can be described by a mean permittivity $\left(\epsilon_{d}=1.6\right)$, with (full line) or without (dashed line) taking into account the increased electron damping due to surface scattering for smaller particles. The inset shows a histogram of transmission changes $\delta T / T$ for one particle size. It demonstrates that indeed single particles and not aggregates are detected. 

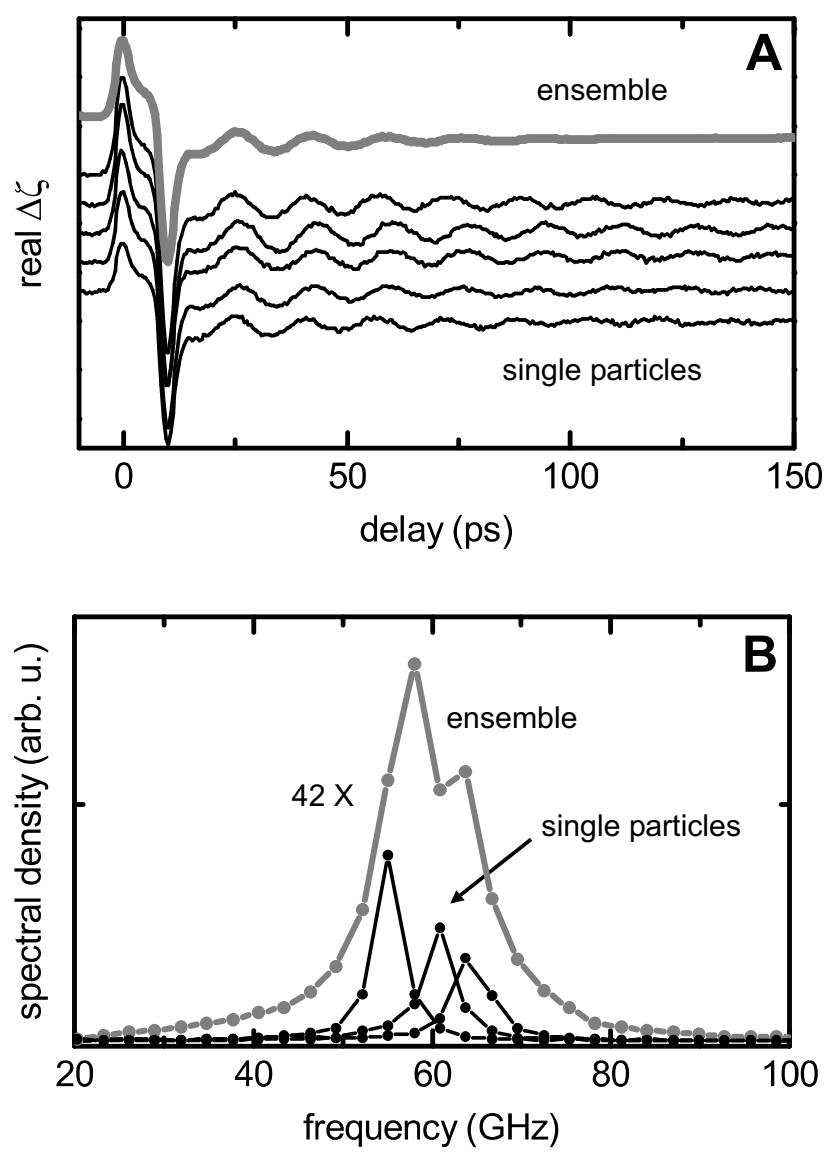

FIG. 5: Comparison of delay scans (left panel) and power spectra of the oscillatory part (right panel) of a set of 29 single gold nanoparticles (examples shown as thin black lines) to a reconstructed ensemble measurement (thick grey line). For this experiment the sample was covered by indexmatching fluid, which increased the damping of oscillations. The particles have a nominal size of $50 \mathrm{~nm}$ with a spread of $6 \%$. The average spectrum in part (B) is scaled by 42 for clarity. The quality factor $Q=\nu / \Delta \nu$ is 4 for the ensemble and about 13 for the individual traces. 

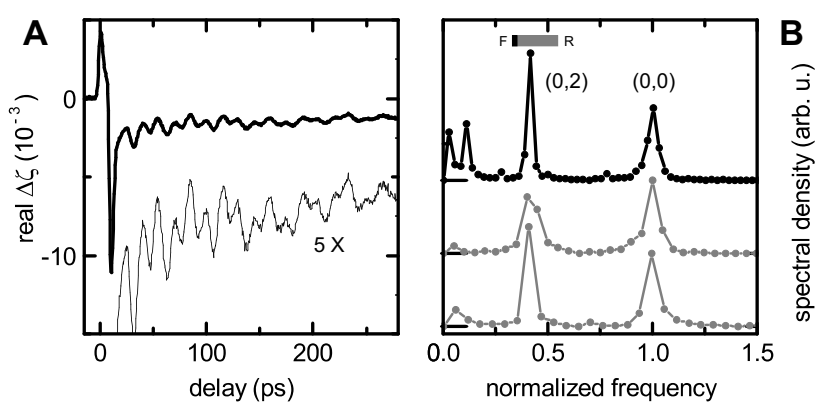

FIG. 6: (A) Delay scan of a single gold nanoparticle. The oscillation pattern shows a complex modulation. (B) Power spectra of this particle's oscillation (top spectrum) and of two other particles. Frequencies and amplitudes are normalized to those of the $(0,0)$ mode (The absolute frequencies were $67 \mathrm{GHz}, 59 \mathrm{GHz}, 63 \mathrm{GHz}$, from top to bottom). The power spectra clearly show, besides the fundamental breathing mode, an additional mode at the same relative frequency to the breathing mode, for each of the three particles. This mode is ascribed to a shear deformation of the particle. The low frequency peak (top spectrum: $28 \mathrm{GHz}$ ) lies between frequencies of the $(0,2)$ mode for free boundary ( $F$, thick line on bar) and for rigid boundary ( $R$, end of bar), calculated according to Lamb's theory of a vibrating elastic sphere [76]. 

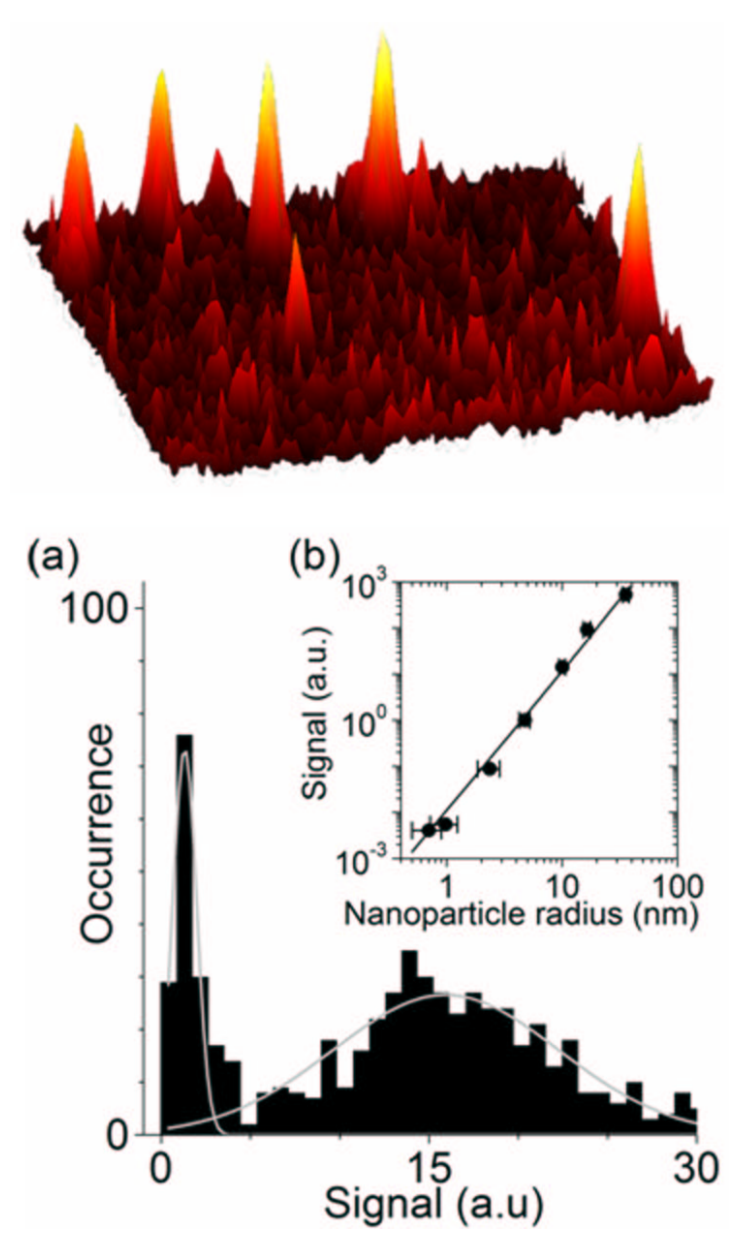

FIG. 7: Top: 3D representation of a photothermal heterodyne image $\left(5 \times 5 \mu \mathrm{m}^{2}\right)$ containing individual gold-clusters of $1.4 \mathrm{~nm}$ diameter. The particles are detected with a relatively small heating power $(\sim 3.5 \mathrm{~mW})$ and a remarkably large signal-to-noise ratio (larger than 10$)$ at an integration time of 10 ms. Bottom: Size dependence of the absorption cross-section of gold nanoparticles with diameters ranging from $1.4 \mathrm{~nm}$ to $75 \mathrm{~nm}$. Samples containing nanoparticles of two different (successive) sizes (1.4 \& $5 \mathrm{~nm}, 2 \& 5 \mathrm{~nm}, 5 \& 10 \mathrm{~nm}$ up to $33 \& 75 \mathrm{~nm}$ ) are prepared. For each sample, a histogram of the signal amplitudes was generated as shown in (a). All the histograms displayed bimodal distributions and the mean of each population was measured. This allows one to calibrate the size dependence of the absorption cross-section normalized to that of $10 \mathrm{~nm}$ particles (b). A good qualitative agreement with a third-order law of the absorption cross-section vs the radius of the particles is obtained (solid line in (b)) as expected by the Mie scattering theory. 

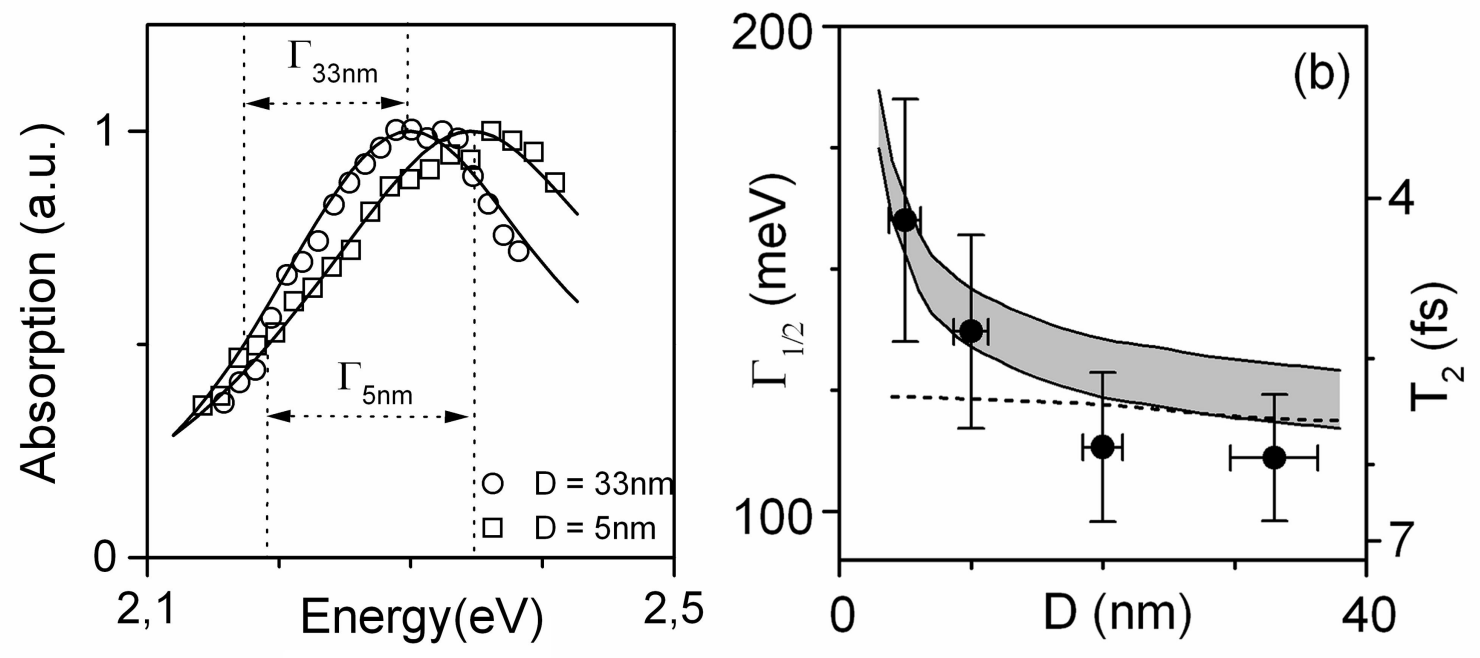

FIG. 8: Left: Normalized absorption spectra of 2 single gold nanoparticles of diameters $33 \mathrm{~nm}$ (gray open circles) and $5 \mathrm{~nm}$ (black open squares). The extracted width at half-maximum is shown on both nanoparticle spectra. The experimental spectra are compared with simulations based on Mie theory (solid lines) using a size-dependent modification in the dielectric constant of gold. Right: Size-dependence of the plasmon resonance width. Experimental data (circles with standard deviations) are compared with Mie theory without (dotted line) and with (gray area) size-dependent correction. The gray area accounts for the experimental uncertainties on the bulk dielectric function of gold given in [62]. 


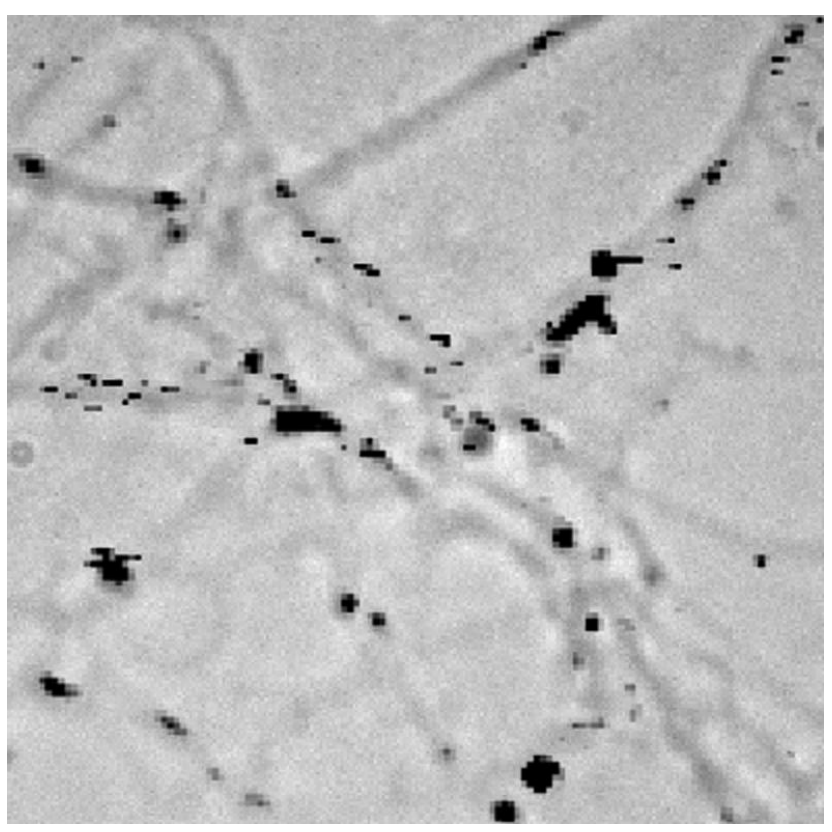

FIG. 9: Superimposed bright field and PHI images of a $20 \mu \mathrm{m} \times 20 \mu \mathrm{m}$ portion of live cultured hippocampal neurons with GluR2 receptors labelled with $5 \mathrm{~nm}$ gold nanoparticles. The PHI image (black spots and stripes) reveal the presence of point-like signals which correspond to immobile GluR2-linked nanoparticles. Most nanoparticles move during the raster scan of the sample, which produces characteristic stripe signals. The excitation intensity is $400 \mathrm{~kW} / \mathrm{cm}^{2}$, the integration time $5 \mathrm{~ms}$ and one pixel is $150 \mathrm{~nm}$ in size. 